\title{
Ventricular Septal Rupture following Acute Myocardial Infarction - A Rare complication
}

\author{
MUHAMMAD MOBAROCK HOSSAIN ${ }^{1}$, SM MOSTAFA ZAMAN ${ }^{1}$, FAKHRUL ISLAM KHALED ${ }^{1}$, \\ MOHAMMAD FAISAL IBN KABIR, NOOR MOHAMMAD ${ }^{1}$, MOHAMMAD ABDUR RAHMAN ${ }^{1}$, \\ ARIFUL ISLAM JOARDER ${ }^{1}$, ANITAMARIUM ISLAM ${ }^{2}$, HARISUL HOQUE ${ }^{1}$
}

\author{
${ }^{1}$ Department of cardiology, Bangabandhu Sheikh Mujib Medical University(BSMMU), Dhaka, Bangladesh, ${ }^{2}$ Department of \\ internal medicine, Apollo Hospitals Dhaka
}

Address of Correspondence: Dr. Muhammad Mobarock Hossain, , Resident, Department of cardiology, Bangabandhu Sheikh Mujib Medical University, Dhaka, Bangladesh, E mail: mobarockdr@gmail.com

Ventricular septal rupture (VSR) after acute myocardial infarction with the consequence of hemodynamic unstability is a rare complication and it's an medical emergency. Mortality of these group patients is higher than $90 \%$ to $95 \%$ without a rapid diagnosis and correction by surgical intervention.Spontaneous closure of VSR is extremely rare. We report the case of a patient with acute myocardial infarction with ventricular septal rupture (VSR) with cardiogenic shock that was diagnosed in our modern coronary care unit by the bed side portable echocardiographic machine (vivid).

The incidence of ventricular septal rupture (VSR) after acute myocardial infarction is extremely rare in this reperfusion era.This condition is associated with a high mortality rate, even after the cardiac surgery . Our case emphasizes the risk factors and evolution of this condition.

A 36 years old young hypertensive young man was admitted on $8^{\text {th }}$ August,2014 through the emergency department of university cardiac centre for central chest pain ,dyspnea, nausea and bilateral shoulder pain for the last last 7-8 days. He consulted with his family physician for the same complaints and later he was referred to get admission in our hospital. The patient's condition was detoriating gradually. Physical examination revealed a regular pulse of 110 beats $/ \mathrm{min}$. The blood pressure was 100/70 mmHg and there was a systolic murmur best heard at the apex, radiating to the axilla. . Pulmonary rales were present and there was no peripheral edema, hepatomegaly and raised JVP.

The 12-lead electrocardiogram Figure 1) showed sinus rhythm at 125 beats/ min, low voltage QRS complex voltage in the limb leads, q waves in II,III, AvF and a $4 \mathrm{~mm} \mathrm{ST}$ elevation in the anterior leads $\left(\mathrm{V}_{2-4}\right)$.

Serum troponin T level at admission was $1.75 \mathrm{ng} / \mathrm{ml}$, CKMB 28, Serun creatinine 1.25, Serum electrolytes are within normal limit.

He was managed as a case of acute antero-septal MI with old inferior MI and on the following day after his admission he developed cardiogenic shock, examination reveals pulse was 115 beats/min and blood pressure was $80 / 40 \mathrm{~mm}$ of Hg.Then we put the patient under ionotrophic support ,transthoracic echocardiography was done and (figure $2,3 \& 4$ ) revealed a small rupture of the apical ventricular septum (figure 2) causing a VSR with left-to-right shunt (figure 3) and Doppler study showed the pressure gradient

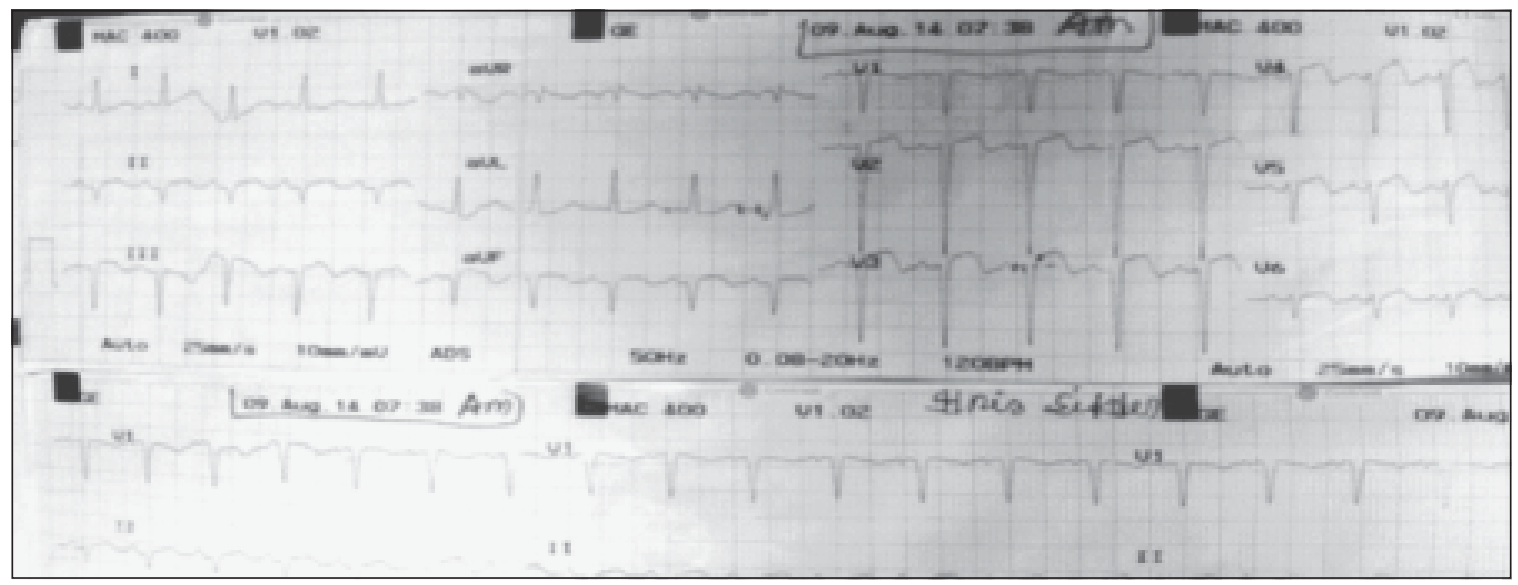

Fig.-1: Electrocardiogram at admission. 


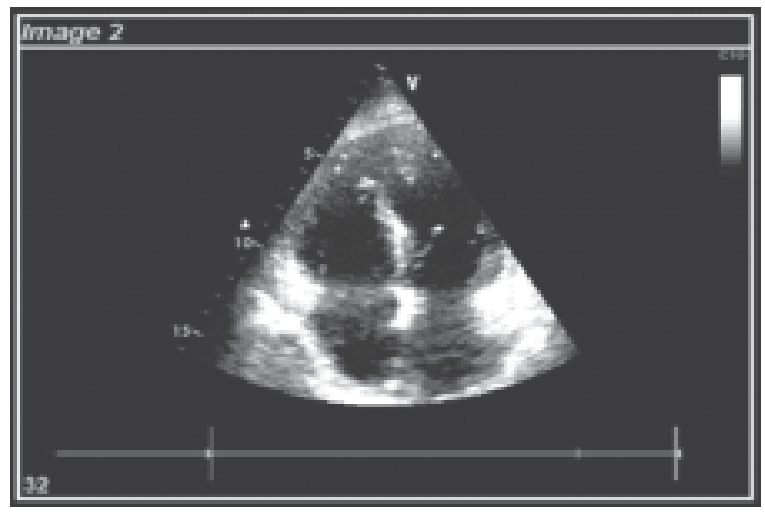

Fig.-2: Revealed a small rupture of the apical ventricular septum

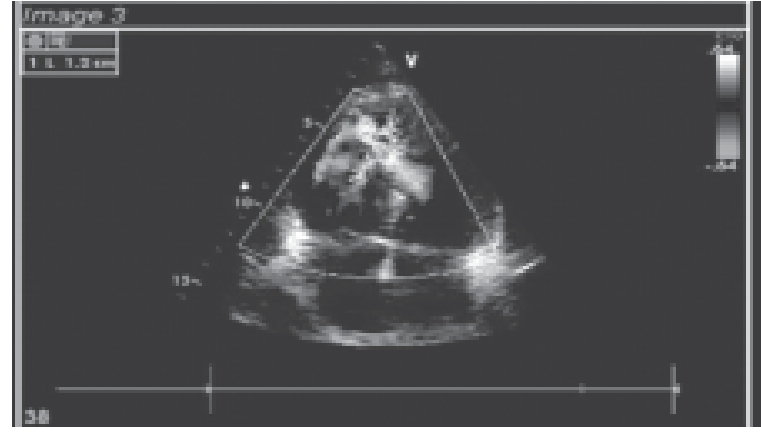

Fig.-3: Revealed a small rupture of the apical ventricular septum (image 2) cusing a VSR with left-to-right shunt

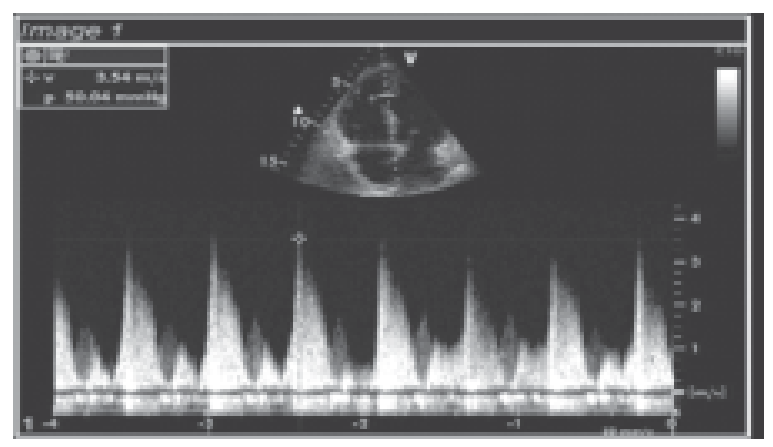

Fig.-4: Doppler study shows the pressure gradient 50.04 $\mathrm{mm}$ of $\mathrm{Hg}$

of $50.04 \mathrm{~mm}$ of $\mathrm{Hg}$ (figure 4) and the size of VSR was $12 \mathrm{~mm}$ (figure 3). The interatrial septum seemed to be intact. Transesophageal echocardiography was not performed.

A diagnosis was made of acute anterior myocardial infarction with old inferior MI complicated by a VSR. For this reason, we refered this case to the cardiovascular surgery department and they advised for conservative management followed by surgical repair of VSR.
The hemodynamic status worsened gradually requiring inotropic support with dopamine and dobutamine along with other anti ischemic therapy. Now the patient is feeling relatively well as there is no chest pain, no nausea, no shoulder pain except generalized weakness. His vitals are as follows. Pulse 105 beats/min, BP 100/80 mm of Hg (with ionotrophic support), respiratory rate 21/min, SPO2-97\%, bilateral basal crepitations present.

\section{Discussion:}

VSR is a rare but serious complication of acute Myocardial infarction that is, in almost all cases, fatal without early surgical intervention. It had an incidence of

$1-3 \%$ in the era before reperfusion therapy, decreasing with the introduction of thrombolytic therapy. ${ }^{1,2}$ VSR is more infrequent than a rupture of the ventricular free wall. Women are affected more commonly than men. Other risk factors are age, non-smoking and hypertension.VSR usually occurs 2-8 days after the infarction and often precipitates cardiogenic shock. There are a few reported cases of silent myocardial infarction followed by an asymptomatic VSR or presenting as chronic congestive heart failure ${ }^{4}$. The size of the defect determines the magnitude of the left-to-right shunt and consequently the hemodynamic deterioration, which affects survival. Complete spontaneous closure of such an acquired defect is extremely rare ${ }^{5 . .}$ There are three types of VSR (the original classification made by Becker and van Mantgem was for free-wall rupture): in type I there is an abrupt tear in the wall without thinning; in type II, the infarcted myocardium erodes before rupture and is covered by a thrombus; and type III represents the perforation of a previously formed aneurysm. ${ }^{6}$ The blood supply to the septum originates from branches of the left anterior descending coronary artery, the posterior descending branch of the right coronary artery, or the circumflex artery when it is dominant. VSR has equal frequency in anterior and nonanterior infarctions. ${ }^{7}$ Anterior $\mathrm{MI}$ is associated

with rupture of the apical septum, in inferior MI, it often occurs at the base of the heart. An MI associated with a VSR is usually extensive. Early treatment of MI with thrombolysis may reduce the incidence of VSR, influence the time to septal rupture and improve the outcome. Development of thrombi in the left ventricle after myocardial infarction is not uncommon. In contrast, right ventricular thrombi are rare. Right heart thrombi may also develop in situ as a result of blood stagnation in patients with a cardiomyopathy or with catheter or pacemaker leads. Floating right heart thrombi in most cases are seen by accident, in transit from deep veins to the pulmonary artery. 
If a patent foramen ovale or atrial septal defect is present, a thrombus can cross from the right atrium directly into the left atrium with paradoxical embolism. The direct demonstration of paradoxical embolism by imaging a thrombus traversing the foramen ovale is unusual. $8,9,10$ Our case illustrates the typical risk factors of VSR: an extensive acute myocardial infarction (anterior, with right bundle branch block) in an old hypertensive, non-smoking woman, with no history of angina. We have described a rare situation in this rare condition-the partial occlusion of the defect by a thrombus — which may have slowed the evolution

\section{References:}

1. Birnbaum Y, Fishbein MC, Blanche C, Siegel RJ. Ventricularseptal rupture after acute myocardial infarction. N Engl JMed. 2002; 347: 1426-1432.

2. Gueret P, Khalife K, Jobic Y, et al. Echocardiographic assessment of the incidence of mechanical complications during the early phase of myocardial infarction in the reperfusion era:a French multicentre prospective registry. Arch Cardiovasc Dis. 2008; 101: 41-47.

3. Crenshaw BS, Granger CB, Birnbaum Y, et al. Risk factors, angiographic patterns, and outcomes in patients with ventricular septal defect complicating acute myocardial
infarction.GUSTO-I (Global Utilization of Streptokinase and TPA for Occluded Coronary Arteries) Trial Investigators. Circulation. 2000; 101: 27-32.

4. Lazopoulos G, Manns-Kantartzis M, Kantartzis M. Giant left ventricular aneurysm and intraventricular septal defect after silent myocardial infarction. Hellenic J Cardiol. 2009; 50: 142-143.

5. Ilia R, Goldfarb B, Wanderman KL, Gueron M. Spontaneous closure of a traumatic ventricular septal defect after blunt trauma documented by serial echocardiography. J Am Soc Echocardiogr. 1992; 5: 203-205.

6. Becker AE, van Mantgem JP. Cardiac tamponade: a study of 50 hearts. Eur J Cardiol. 1975; 3:349-358.

7. Batts KP, Ackerman DM, Edwards WD. Post infarction rupture of the left ventricular free wall: clinicopathologic correlates in 100 consecutive autopsy cases. Hum Pathol. 1990;21: 530-535.

8. Meacham RR, Headley AS, Bronze MS, Lewis JB, Rester MM. Impending paradoxical embolism. Arch Intern Med. 1998; 158: 438-448.

9. Avery EG, MacGillivray TE. Arrested paradoxical emboli in transit diagnosed by intraoperative transesophageal echocardiography. Anesth Analg. 2002; 95: 1569-1571.

10. Srivastava TN, Payment MF. Paradoxical embolismthrombus in transit through a patent foramen ovale. N Engl J Med. 1997; 337: 681. 